\title{
Tau-leaped Particle Learning
}

\author{
Jarad Niemi*1 and Michael Ludkovski ${ }^{2}$ \\ ${ }^{1}$ Iowa State University, Ames, IA, USA; ${ }^{2}$ University of California, Santa Barbara, Santa Barbara, CA, USA
}

\section{Objective}

Develop fast sequential Bayesian inference for disease outbreak counts.

\section{Introduction}

Development of effective policy interventions to stem disease outbreaks requires knowledge of the current state of affairs, e.g. how many individuals are currently infected, a strain's virulence, etc, as well as our uncertainty of these values. A Bayesian inferential approach provides this information, but at a computational expense. We develop a sequential Bayesian approach based on an epidemiological compartment model and noisy count observations of the transitions between compartments.

\section{Methods}

For simplicity, consider an SIR epidemiological compartment model where compartments exist for susceptible, infected, and recovered individuals. Transitions between compartments occur in discrete time with transitions numbers given by Poisson random variables, the tau-leaping approximation, whose means depend on the current compartment occupancy and some unknown fixed parameters, e.g. virulence. Binomial observations, with possible unknown sampling proportion, are made on these transitions.

The standard sequential Bayesian updating methodology is sequential Monte Carlo (SMC), a.k.a. particle filtering. The original bootstrap filter is effective when the system has no fixed parameters, but exhibits marked degeneracy otherwise [1]. An approach based on resampling the fixed parameters from a kernel density estimate provides a generic approach with less degeneracy [2].

We build methodology based on a particle learning framework [3]. In this framework, each particle carries a set of parameter-specific sufficient statistics and samples parameter values whenever necessary. In addition, the methodology promotes a resample-move approach based on the predictive likelihood that reduces degeneracy in the first place.

An improvement on the particle learning framework in this model is that some fixed parameters can be integrated out of the predictive likelihood. This Rao-Blackwellization provides an SMC methodology with reduced Monte Carlo variance.

\section{Results}

For a fixed number of particles or computational expense, we show improvements in accuracy relative to the kernel density approach and an alternative approach based on sufficient statistics [4] where compared with a gold-standard Markov chain Monte Carlo analysis.

\section{Conclusions}

Many surveillance systems collect counts of adverse events related to some disease. These counts are expected to be a fraction of the true underlying disease extent. The methodology developed here allows a fully Bayesian analysis that uncovers the true number of infected individuals as well as disease virulence based on these count data. This statistical approach can be combined with an optimal policy map to help public health officials react effectively to initial disease reports.

\section{Keywords}

surveillance; Bayesian; sequential Monte Carlo; particle learning

\section{References}

[1] Gordon, Salmond, and Smith. Novel approach to nonlinear/nonGaussian Bayesian state estimation. IEE Proceedings Part F: Communications, Radar and Signal Processing. 140(2): 107-113 (1993).

[2] Liu and West. Combined parameter and state estimation in simulation-based filtering. Doucet, De Freitas, and Gordon, ed. Sequential Monte Carlo Methods in Practice. Springer-Verlag, New York. 197217 (2001).

[3] Carvalho, Johannes, Lopes, and Polson. Particle learning and smoothing. Statistical Science. 25(1):

88-106 (2010).

[4] Storvik. Particle filters in state space models with the presence of unknown static parameters. IEEE Transactions on Signal Processing. 50(2): $281-289$ (2002).

\section{*Jarad Niemi}

E-mail: niemi@iastate.edu 